\title{
Variáveis semióticas do espaço na cultura de meios ${ }^{1}$
}

Irene Machado

Resumo: Seguindo os pressupostos da semiótica da cultura segundo os quais "todos os meios e processos de comunicação não são apenas potencialmente criadores de espaço, como também definem a natureza espacial dos seres que nele interagem", examina-se aqui o espaço semiótico modelizado pelas variações de códigos linguísticos e dos meios elétricos de comunicação. Chega-se assim à configuração do espaço ressonante como variável de espaço na tecnocultura.

Palavras chave: espaço semiótico; ressonância; visualidade; meios; fronteira; geopolítica.

Abstract: Semiotic variables of space in media culture - Following the assumptions of semiotics of culture according to which "all media and communication processes are not only potentially creators of space, but also define the spatial nature of beings interacting in it", we examine here the semiotic space generated by modeling variations of linguistic and electrical codes of media. We then achieve the resonance space configuration as a variable of space in technoculture.

Key words: semiotic space; resonance; visuality; media; boundary; geopolitics.

\section{Introdução}

Quando situamos a preocupação central deste trabalho - a relação entre comunicação e espaço - no âmbito daquilo que Hall denominou de proxemia, isto é, dos estudos dedicados ao "uso que o homem faz do espaço como elaboração especializada da cultura" (HALL, 1977, p. 13), somos desafiados a esclarecer de que tipo de elaboração especializada tratamos aqui.

1 Trabalho apresentado no encontro do GP Semiótica da Comunicação por ocasião do XXXVII Congresso Brasileiro de Ciências da Comunicação, Foz de Iguaçu, 2014, como resultado parcial das investigações realizadas dentro do Projeto de Pesquisa Espaço Semiótico nos Meios de Comunicação: perspectivas, frequência, ressonância (CNPQ, PQ 2013-2015), destinado a examinar a amplitude da noção de espaço semiótico no cenário dos meios de comunicação em sua versão de meios tecnológicos (ver MACHADO, 2012; 2014a). 
Afinal, o tema de nossa investigação segue a linhagem dos trabalhos "em que a comunicação constitui o núcleo da cultura" (ibid., p. 13). Todavia pesa nessa definição pelo menos uma distinção, a saber: na cultura, comunicação constitui um conjunto de manifestações não limitadas à troca linguística em torno da qual gravitam os demais sistemas culturais. Integram esse conjunto diferentes interações com o ambiente efetivadas com meios e agentes mediadores de natureza técnica. Nas interações, signos realizam ações não apenas de troca como também de ampliação de informação em circuitos de possibilidades comunicativas.

Com isso, os mediadores adquirem o poder de definição sobre o uso cultural do espaço introduzindo uma dinâmica distintiva de relações em que a multiplicação dos códigos, favorecida pelo aprimoramento dos meios técnicos, torna-se um potencial processador tanto de linguagens quanto de sistemas de cultura, como é o caso do espaço.

Em torno desse pressuposto se desenvolvem campos de estudo em que a problematização do espaço se complexifica tanto em sua natureza (SANTOS, 2006), quanto em sua construção como lugar de informação (FERRARA, 1990). Em nome de uma dinâmica assim configurada, não apenas o uso do espaço como também os modos operacionais desse uso se tornam os principais agentes de transformação. No limite, o espaço é apreendido no movimento de suas transformações.

Diríamos, pois, que esse estudo está inserido no campo das pesquisas sobre comunicação e cultura, contudo, o problema de sua inquietação emerge das transformações ocorridas quando da ampliação dos códigos culturais cujo raio de ação colocou em evidência não apenas a diversidade de seus espaços como também as variáveis de sua constituição. Vale esclarecer que o termo "variável" comparece aqui na sua acepção matemática: elemento que ao assumir diferentes valores projeta gradiente de distintas configurações.

Nesse sentido, o valor em uma variável não é propriedade, mas condicionante, caso que entendemos ocorrer com as transformações a que se submete o espaço diante dos diferentes sistemas de signos da cultura, ainda que no momento o alvo do debate recaia sobre as variáveis desenvolvidas a partir dos meios da comunicação eletro-eletrônica. Quando se tem no horizonte as variáveis semióticas não se trata apenas de focalizar as mudanças ou variações do ou no espaço, mas de alcançar a dimensão de valor que o espaço configura.

Assim, no contexto do uso visual da cultura alfabética, às variáveis do espaço consagradas pelo registro da escrita se contrapõem as variáveis do espaço dos circuitos elétricos. Ainda que haja implicação mútua entre os diferentes sistemas, há de se ponderar sobre os sistemas de signos em disputa na produção de espaços culturais, uma vez que os circuitos elétricos submetem os espaços à ressonância de suas variáveis.

Emerge uma concepção de espaço cuja configuração não decorre do registro nem da dominância visual, mas da observação das variáveis resultantes de movimentos 
sensoriais baseados em vibração e reverberação. Em vez de uma determinação dominante - caso da visualidade - o que se observa é uma constante transformação e intervenção a partir dos códigos instituído pelos diferentes meios.

Noções como a de lugar passam a demandar um refinamento de acepção, sobretudo porque do ponto de vista da ressonância, as topografias passam a disputar espaço com topologias de variáveis relacionais e associativas. No limite, diríamos que o uso qualificado do espaço se torna modelização de códigos sempre renovados pelos meios de comunicação em expansão. Se o uso do espaço implica modelização de códigos culturais, as variáveis do espaço revelam seu caráter semiótico. Tal é a hipótese fundamental de nossa investigação cujos argumentos se espera desenvolver na sequência.

\section{Uso cultural do espaço como processo e problema semiótico}

Ao reconhecer a ressonância como variável de espaço graças ao uso que os meios de comunicação realizam de códigos elétricos, os estudos sobre o espaço semiótico não afirmam suas premissas com base em negativas. Descartemos, pois, a oposição entre visualidade e ressonância ou imagem e sonoridade. Tratemos, sim, dos movimentos controvertidos que os sistemas de signos desenvolvem nos espaços culturais de modo a produzir variáveis não suscetíveis de previsibilidade, tais como se observou nas ocorrências da ressonância como variável cultural de espaço.

Ainda que advindo de processos relacionados à produção sonora, o conceito de ressonância ultrapassa seus limites para modelizar o movimento de reverberação em circuitos vibratórios, fazendo emergir topologias. Em vez de lugares posicionados, busca alcançar as fronteiras entre limiares e passagens. Em nossos estudos, observamos a configuração do espaço ressonante em diferentes experiências culturais, a começar pela necessidade antropológica de transformar as relações com o entorno em manifestação comunicativa de linguagem. Afinal, como se pode ler no argumento de Schlögel, como seres sensoriais que somos, nosso comportamento no mundo espacializa relações de formas distintas e inusitadas.

Porque somos seres espaciais também vemos espacialmente. «Algo» sempre tem superfície, profundidade, cor, movimento, odor. Tudo transmite algo: distância, proximidade, pressa, lentidão, certeza, excitação ou sossego. Se contemplamos uma praça, sempre é do ponto de intersecção de movimentos em diversas direções. (SCHLÖGEL, 2007, p. 52)

É próprio da ressonância o movimento em outras direções, externas a qualquer continência e, particularmente, imprevisível. Tal particularidade foi possível examinar em outro estudo (MACHADO, 2014c) a propósito da própria expansão das línguas nacionais derivadas do latim em seu deslocamento do continente europeu rumo ao além-mar 
desconhecido. Observou-se que a expansão ultramarina, ocorrida entre os séculos XV e XVI, criou vínculos estreitos entre domínios topográficos, linguísticos e políticos.

Contudo, a mentalidade cartográfica reinante desenhou uma superfície gráfica consonante com interesses políticos imediatos, o que levou muitos estadistas e governantes a crer que a geopolítica deveria ser a consciência geo-gráfica do Estado (MAGNOLI 1990, p. 84). O próprio Cristovão Colombo, antes de se tornar navegador, era cartógrafo, habituado, pois, a deslocar-se no espaço a partir da noção de linhas, retas e planos.

Ainda que a variável visual da cultura letrada em voga seja dominante, impossível ignorar o papel das variáveis que sustentam a concepção de espaço para um contexto de ressonância, inclusive, da própria língua: as línguas nacionais europeias não ficaram imunes às variantes continentais americanas. Somente por imposição de dominantes sócio-históricas e políticas bastante limitadas se consagrou a restrição da espacialidade em função de topografias visuais, ignorando-se as topologias articuladas por ressonâncias e variáveis semióticas do espaço.

O exemplo histórico que se reporta à aventura cultural do encontro linguístico em deslocamento geopolítico contrasta, assim, com a experiência científica que focaliza o viés da expansão que construiu a variável tecnológica do espaço ressonante o qual delimitou as bases da história dos meios bem como os fundamentos de sua ontologia. Estamos nos referindo às descobertas desenvolvidas no contexto das gravações elétricas que abriram caminho para a emergência de princípios construtivos do espaço ressonante em processos audiovisuais, particularmente a kinesis tornada movimento no cinema e nos meios sonoros.

Experiências históricas realizadas por cientistas tais como Thomas Edison e Graham Bell deixaram um legado cujos lastros conceituais se tornaram a pedra fundamental de conhecimento na área (STERNE, 2003). O experimento que procurou registrar o som numa superfície, inicialmente em cilindros e, depois, em discos e fitas eletromagnéticas, revelou uma ocorrência inusitada, muito diferente de um mero registro elétrico. No processo de gravação, o "som" tomado na partida é convertido em circuitos elétricos para depois ser transmitido e recebido enquanto "som". Do ponto de vista acústico, há todo um movimento de interferência processual de modo a conservar a natureza do fenômeno sonoro.

Tal interferência coloca entre a codificação e a descodificação a importância de um processo técnico de recodificação que foi definido como "transdução" (ibid.). Além de potencializar no espaço semiótico diferentes níveis de codificação, a transdução evidencia o papel epistemológico do espaço gerado pelo meio tecnológico. Como ignorar entre a enunciação, a operação técnica e a percepção níveis distintos de realização acústica? A transdução sinaliza para um movimento imprevisível que se encaminha para além das variáveis envolvidas.

Ainda que tenha sido conceptualizado a partir da eletricidade e do processamento da informação biológica, como examinamos em outro momento (MACHADO, 2011), a transdução coloca em evidência modelizações culturais com o entorno, 
mesmo em interações mais elementares, caso das interações de propriocepção (ver mais adiante), o sensorialismo da Umwelt (UEXKÜLL, 2001) ou as interações que levaram à linguagem no processo evolutivo (SEBEOK, 1995).

Ainda que citados de modo esquemático, os exemplos históricos se colocam no horizonte especulativo do debate sobre o espaço ressonante, visto que dimensionam a ontologia dos meios, particularmente, em sua versão audiovisual que não se constrói senão a partir da transdução do movimento, seja do som, seja da imagem, nas mais variadas formas de sua constituição.

Em suas observações sobre a experiência do cinema, Jakobson elaborou análises memoráveis a respeito. Num contexto em que se amputava da análise fílmica a composição acústica, Jakobson enfatizava a ressonância da montagem contra noções que preconizavam a autonomia visual da "fotografia em movimento" em que a reprodução da fala sinalizava "decadência" (JAKOBSON, 1970, p. 155-156).

Para Jakobson, posturas como essas denunciavam uma total ignorância com relação ao "objeto óptico-acústico do cinema". Seus exemplos não poderiam ser mais eloquentes. Enquanto no cinema silencioso o som era traduzido em planos e figurações de objetos sonoros, no cinema sonoro, a gravação do som não podia prescindir da transdução, visto que a gravação magnética precisava ser recodificada em termos ópticos para, somente depois, durante a projeção, ser recodificada em emissão sonora. Todo esse processo modelizante acompanha a latitude da definição audiovisual do cinema e do espaço ressonante que ele introduz enquanto manifestação da tecnocultura.

Justifica-se, assim, a necessidade de adesão aos investimentos que insistem na aproximação de noções em que o espaço ressonante situa em seus fundamentos vetores espaciais constituídos por circuitos e frequências segundo padrões de diferentes intensidades. Além de modelizar o espaço fora das coordenadas visuais, abre-se caminho para o desenvolvimento de um repertório crítico de análise e de compreensão no quadro de qualidades diferenciais.

Tanto a noção de espaço semiótico quanto a de espaço ressonante se apresentam como resposta a demandas sobre a elaboração de parâmetros qualificados para análise das variáveis culturais dos sistemas tecnológicos. Disso se convenceu McLuhan quando, ao entender a natureza da informação elétrica, dimensionou uma outra forma de organização do espaço que ele definiu como espaço acústico ressonante (MCLUHAN; POWERS, 1996). Com base nessa noção é que foi possível aos estudiosos observar a natureza das expansões sensoriais dos meios acústicos e audiovisuais no contexto sígnico de sua constituição.

Dado o caráter distintivo do espaço ressonante e de seus circuitos criadores de topologias dinâmicas, somos desafiados a refazer a pergunta inicial: quais são as bases da interação entre comunicação e cultura quando a ressonância se converte em dimensão constitutiva do espaço?

Para examinar tal questão, recorremos aos estudos sobre o espaço semiótico formulado por Lótman, particularmente sua compreensão da semiose como processo 
de transformação espacial e temporal orientado não somente por variações como também pela geração do imprevisível, manifestação imponderável da inteligência do sistema.

Ocupado em dimensionar a dinâmica processual do espaço, Lótman propõe uma premissa teórica precisa: o conceito de semiosfera. No horizonte de tal concepção o uso cultural do espaço pode ser dimensionado segundo as qualidades diferenciais da semiose em sistemas envolvidos. Com isso, o processo de expansão é flagrado do ponto de vista evolutivo, isto é, em seus embates e lutas: um signo não se define porque ocupa o lugar de outro mas sim porque se mostra em confronto, em luta pelos elementos que possam evidenciar os aspectos distintivos da semiose espacial emergente. ${ }^{2}$

\section{Espaço semiótico e luta pela informação}

Se existe uma chave conceitual para fundamentar a noção de espaço semiótico sem dúvida ela tenderá para o movimento e, particularmente, sua expressão como continuidade, o continuum semiótico que organiza as relações em espaços culturais. Com isso, livra-se do risco de atribuir aos sistemas de signos o caráter de totalidade ou continência num campo visual. Os sistemas culturais são tomados na continuidade e contingência o que implica uma dinâmica de luta muito mais acirrada ante ameaças de acomodação.

Para Lótman, a luta pela informação $(1978 ; 1985 ; 1990)$ concentra o núcleo essencial da relação entre cultura e espaço. Em sua luta pela informação, os distintos constituintes do espaço se tornam signos quando codificados, isto é, quando transformados e modelizados pela ação de signos e linguagens em sistemas culturais.

O mecanismo da modelização que permite alcançar o espaço na dinâmica das relações, dentro e fora dele, qualifica o entendimento de Lótman e introduz o viés particular da "virada semiótica do espaço" no debate que, desde os anos de 1960, promovem a virada espacial. Nele o mundo dos objetos é transformado em mundo de signos (LÓTMAN 1990, p. 54). Ao povoar o espaço com signos, criam-se dispositivos constitutivos do espaço semiótico de interação: a fronteira é, senão o mais importante, pelo menos o mais problematizador dos contatos e das ações de uns sobre os outros. O conceito semiótico de fronteira desloca a noção de espaço da superfície topográfica para os ambientes multidirecionais das topologias onde a própria coordenada temporal atua como agente transformador.

Em vez de lugar de informação, Lótman observa transformações no tempo. Com isso alcança os sistemas culturais em diferentes desenvolvimentos e prospecções. É o que, num outro contexto de reflexão, historiadores observaram como espaço ressonante na cultura acústica oral-aural e tecnológica (MCLUHAN; POWERS, 1996; ONG, 1967; STERNE, 2003; ERLMANN, 2010), base fundamental dos estudos denominados de proxêmica,

2 Ver ainda o trabalho de Santos (2006) em que a tecnosfera fundamenta a ontologia do espaço; e os estudos de Ferrara sobre a semiótica visual do espaço (FERRARA, 2002). 
que não pode ser ignorada quando se trata de dimensionar a semiótica do espaço (HALL, 1977).

Embora tenha formulado conceitualmente a noção de um espaço semiótico amplo e culturalizado, Lótman não viveu o suficiente para acompanhar as explorações tecnológicas criadoras do espaço semiótico cuja ressonância evocaria as tramas semiósicas da semiosfera. Contudo, nada nos impede de perseguir os encaminhamentos possíveis nessa direção, sobretudo quando o debate sobre fronteiras e ressonância se coloca na ordem do dia, subsidiando movimentos especulativos a respeito do papel dos meios na virada espacial consagrada pela tecnocultura.

\section{Limites e limiares e fronteiras}

Se a luta pela informação concentra a relação entre espaço e cultura, há que se sopesar embates entre os estados de natureza e cultura.

Sob o domínio de estados sensoriais extremamente aguçados, certos organismos que vivem em estado de natureza desenvolvem um sistema de percepção do espaço cujo dispositivo de alerta se encontra em prontidão permanente. Além de cobrir continuamente o espaço com uma visão de $360^{\circ}$, desfrutam das intensas relações que fazem das coordenadas espaciais conjuntos exponenciais de vibrações que podem ou não ser apreendidas.

O fato é que os sobressaltos e ameaças podem vir, não apenas de todos os lados, como também com intensidades distintas. Para um sistema perceptual assim organizado, a distinção entre o espaço de um e o espaço do outro só ganha peso e significação em situações de confronto e de luta quando o alvo da defesa é a própria pele no âmbito de um espaço atual.

Nesse caso, a pele torna-se núcleo da propriocepção dos constituintes espaciais carregados de qualidades ambientais. Nela se imprime a capacidade de transposição de limites de modo a desenvolver-se em espaços de liminaridade. Por conseguinte, tanto a convivência quanto a sobrevivência dependem de um modo de viver em consonância com a plenitude multidirecional do espaço e a prontidão sensorial sempre atualizada. Tudo leva a crer que o espaço assim vivenciado desconhece as realizações de cultura que distinguem e separam o que é próprio e o que é alheio para além da superfície da pele.

Aos seres que desenvolvem linguagem coube um outro desfrute do espaço. Em vez de orientação sensorial, baseada em proprioceptores, o usufruto do espaço se realiza, sobretudo, pelo viés de códigos que organizam o espaço com variáveis traduzidas sob forma de conhecimentos, relações e domínios de forças sociais e políticas. Por um lado, estabelece-se a diferença entre o que é próprio e o que é alheio; por outro, luta-se para transgredir limites, colocando fronteiras em xeque. Limites, fronteiras, trânsitos de um lugar para o outro e encontros tornam-se os termos da definição do espaço como estado de cultura em movimentos e limiares, o que sugere a Lótman a própria condição antropológica do homem. Segundo seu entendimento, 
Toda atividade do homem como homo sapiens está ligada a modelos classificatórios de espaço, a sua divisão entre "próprio" e "alheio" e à tradução dos variados vínculos sociais, religiosos, políticos, familiares, à linguagem das relações espaciais. (LÓTMAN, 1996, p. 83)

Na base da distinção entre próprio e alheio se erguem oposições como cultura e não-cultura; cultura e natureza; civilização e barbárie definidoras de estados de cultura de diferentes graus de intensidade. Por isso, ainda segundo Lótman,

[...] todos os tipos de divisão do espaço formam construções homomórficas. A cidade (= porção povoada) se opõe ao que se encontra para além de seus muros (bosques, a estepe, a aldeia, a Natureza, o lugar onde habitam os inimigos), como próprio, o fechado, o culto e seguro, ao alheio, aberto, inculto. Desse ponto de vista, a cidade é parte do universo dotada de cultura. Contudo, em sua estrutura interna, ela copia todo o universo, tendo seu espaço "próprio» e seu espaço «alheio». (lbid., p. 84)

O estudo da tipologia da cultura orienta o entendimento do espaço, sobretudo, porque conta com algumas variáveis perturbadoras quando se trata de considerar a dinâmica dos contatos e das relações culturais no que se refere ao confronto estabelecido entre seus limites e fronteiras adiante da dinâmica de seus limiares.

Desde que as línguas se tornaram os agentes fundamentais na definição de uma cultura e desde que o diálogo se converteu em seu principal mecanismo de funcionamento, a variável mais desestabilizadora das configurações do espaço diz respeito ao mecanismo semiótico da amplificação dos sistemas comunicativos decorrentes dos processos de expansão da linguagem verbal.

Continuando a reflexão iniciada anteriormente a respeito da expansão da língua latina, observa-se que a escalada das línguas no contexto da ocidentalização do planeta tanto se encarregou de instituir as divisões dos Estados nacionais quanto de avançar e romper limites, projetando-se para o além-mar radicalmente outro. A expansão ultramarina cumpriu, a um só tempo, a ampliação dos limites geográficos e linguísticos, vinculando, definitivamente, os domínios geopolíticos ao espaço semiótico da cultura, marcando a experiência histórica de tudo que vive sob fronteiras.

Se o ponto de partida foi a variação, o que se observa é que no processo emergiram variáveis imprevisíveis. Com a descoberta de novos continentes, processou-se uma informação nova. Para além do próprio e do alheio vimos emergir a fronteira de um espaço projetado em função de interesses políticos de dominação e, por conseguinte, tensionado pelo encontro com o outro.

Espaço aqui não enfatiza o lugar, mas o "limiar", consolidando a fronteira como espaço ambivalente: une e separa o próprio e o alheio. Nesse sentido, "a duplicação do mundo na palavra e do homem no espaço forma o dualismo semiótico de partida" 
(ibid., p. 85). Por conseguinte, os homens com suas linguagens e culturas transitam de um lugar para o outro, instauram confrontos no interior dos limites tensionando as linhas que só aparentemente separam o próprio do alheio com diferentes graus de intensidade.

Ao colocar a ênfase no encontro em confronto, tanto no interior de uma cultura quanto entre diferentes culturas, o conceito de fronteira é dimensionado semiótica e culturalmente e não apenas pela divisão geopolítica. Na verdade, a própria definição geopolítica passa a ser entendida como dinâmica de um espaço cultural semiotizado pelas línguas expandidas e por diferentes sistemas de signos da comunicação cultural desenvolvidos no ocidente, sobretudo, a partir da escrita alfabética.

Ao continuum das relações espaciais, os sistemas de signos da cultura opõem e deixam emergir confrontos, o que sugere a configuração de uma singular dinâmica espacial da semiosfera. Nela tanto os seres quanto suas construções dinamizam fronteiras em níveis de distintas constituições, direções e temporalidades, orientados que são pelos encontros culturais. Quanto mais diversificados se revelam os encontros e quanto mais variáveis perturbadoras em confronto, mais complexo se torna o espaço. Imerso na semiosfera, o espaço semiótico assume o funcionamento dinâmico de um sistema liminar. Os encontros culturais por sua vez definem a fronteira como episteme fundamental dos estudos das relações entre comunicação e espaço uma vez que orientado pelo estado sempre disposto de luta pela informação.

\section{Fronteira no âmbito da virada espacial}

As inquietações em torno das transformações do conceito de fronteira não são privilégio do estudo de Lótman, ainda que sua compreensão do espaço semiótico do ponto de vista da semiosfera seja sua premissa epistemológica mais desafiadora.

Nos trabalhos que marcaram a chamada "virada espacial" (BODENHAMER, 2010) nas ciências humanas, desde os anos de 1960, particularmente na geografia, os elementos semióticos consagrados pelas linhas e limites tornaram-se o epicentro da revisão conceitual, sobretudo porque nelas se vislumbram os movimentos e confrontos sociais desencadeados por práticas humanas como formas de intervenção em espaços, como procuramos analisar em outro estudo (MACHADO, 2014a).

Com isso, aquilo que está na margem deixa de ser dimensionado ponto de uma extremidade que divide e separa o centro da periferia. Estar à margem pode não significar estar preso a um lugar nem permanecer do lado de fora, mas sim poder mudar para o outro lado e se movimentar livremente em limiares. Por conseguinte, como nos alerta Houtum, passamos a usar "border as a verb in the sense of bordering"3 ${ }^{3}$ (HOUTUM, 2005, p. 672), problematizando as noções consagradas em que fronteira (border) define

3 Em português a tradução correspondente seria: "fronteira como um verbo no sentido de fazer a margem ou margear" (HOUTUM 2005, p. 672). 
delimitação, espaço demarcado, em distinção a limite (boundary) que enfatiza o espaço de relação e construção social entre diferentes culturas (ibid.).

Ao reconhecer que no centro de gravidade do conceito de fronteira acontecem movimentos que modificam o entendimento das práticas sociais de convivência em espaços, é todo um campo conceitual que passa a reivindicar novos enfrentamentos. Com isso, ainda segundo Houtum, "borders are now pre-dominantly critically investigated as differentiators of socially constructed mindscapes and meaning"4 (ibid., p. 673).

Sem dúvida, o grande impacto de tal entendimento recai sobre o espaço geopolítico modelizado pelos meios em suas diferentes versões tecnológicas (alfabéticas, eletrônicas, digitais). Os meios configuram o vínculo entre comunicação e espaço enfatizando, por um lado, relações de poder e jogos de interesse; por outro, determinação tecnológica. Tudo convivendo em espaço de fronteira.

Disputas por territórios se confundem com disputas pelo controle de sistemas tecnológicos da comunicação em que o controle sobre cabos, redes, fibra óptica, satélites e de bancos de dados evidenciam apenas a ponta de um complexo jogo de poder que se desenrola para muito além da superfície terrestre. Entram em cenas novos agentes semióticos, como observou Price ao afirmar que:

O mundo está engajado num vasto remapeamento de relações do Estado com as imagens, mensagens e informações dentro de suas fronteiras. (...) Tudo está em construção, gerando um abalo profundo e remodelando todos os sistemas de comunicação. (PRICE, 2002, p. 4)

Um remapeamento do mundo em função não somente dos limites estabelecidos por jogos de forças, mas, sobretudo, pela complexidade das relações com o entorno em sua progressiva geração de sistemas comunicacionais, o que mostra uma significativa transformação no próprio repertório da mudança, a começar pelo aparelhamento sociocultural do espaço pelos meios.

$\mathrm{Na}$ incorporação de um termo como "invisibilidade", flagra-se a aproximação de um espaço distinto da geografia. A nova ação geopolítica opera mais incisivamente com linhas imaginárias, limites invisíveis, ubiquidades. E é pelo viés da invisibilidade que alcança o elo que vincula o espaço geopolítico com os meios audiovisuais, eletrônicos e digitais, ambos atuantes no espaço de circuitos e frequências. Não poderíamos ter melhor chave conceitual para introduzir a problemática do espaço semiótico da semiosfera em sua tradução pelas rotas ressonantes da mundialização, globalização e planetarização, sobretudo na versão de uma leitura crítica do espaço.

Vale destacar aqui a força conceitual do repertório crítico fundamentado por uma ampla base semiótica. Para os estudiosos da virada espacial, o espaço geográfico é,

4 "fronteiras são agora predominante e criticamente investigadas como diferenciadores de memória e de sentido social" (ibid., p. 673). 
antes de mais nada, espaço de informação codificada, entendido como um campo contínuo de superfícies projetadas em signos dos diferentes sistemas de referência. A partir da qualidade gráfica desse espaço - traços, letras, números, escalas, formas geométricas, sistemas de cores -, define-se a qualidade da informação nos signos pelos quais a informação se culturaliza.

A introdução de novos signos de projeção do espaço leva a um refinamento cognitivo desse espaço. Das lentes de lunetas aos satélites e dispositivos digitais de teledetecção, o espaço de informação sofre por transformações constantes. Ainda que continuemos a chamar de mapas, o espaço configurado por câmeras e satélites não se limita ao campo geográfico, pelo contrário, qualquer imagem produzida por sensoriamento remoto mostra um espaço orientado para o alto e pelo exterior em relação à superfície do solo. Incorpora-se uma dinâmica ressonante na projeção e o espaço pode ser apreendido por meio de ondas vibratórias e frequências.

Impossível ignorar que vivemos num universo ressonante quando sabemos da quantidade de satélites que gravitam na órbita do planeta para atender às mais distintas demandas.

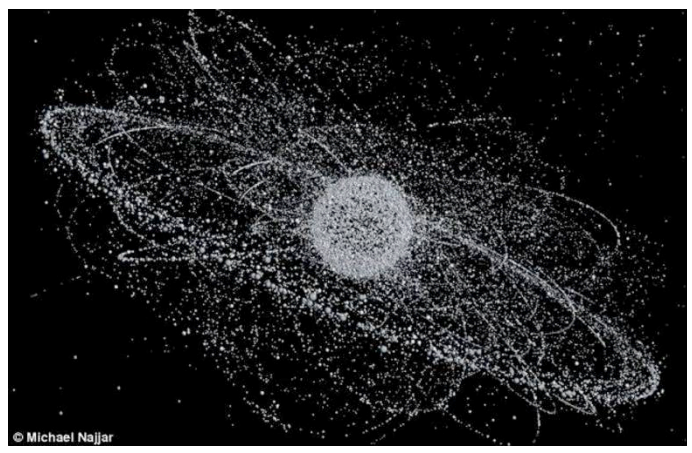

Fig. 1. Fotografia de Michael Najjar - Cada ponto branco representa um dos 370 mil objetos já lançados na órbita da Terra. Fonte: http://www.michaelnajjar.com/images/intro/1.jpg (Último acesso 07/07/2014)

Tal como as línguas culturalizaram os espaços ultramarinos, os meios da comunicação tecnológica culturalizam os espaços por onde as ondas eletromagnéticas e os sinais de redes informáticas e de cabos se movimentam. E é pelos meios atualizados em satélites e antenas que os espaços geopolíticos passam a ser configurados, evidentemente não sem a liderança linguística da língua geopoliticamente dominante. Nenhum sensoriamento do espaço ressonante pode ser pensado à margem do espaço semiótico em que línguas e linguagens informáticas cumprem a tarefa de modelizar os sistemas culturais em relação na semiosfera.

Se o sistema alfabético da escrita permitiu a confecção de sistemas espaciais de referências com signos gráficos tais como desenhos, números, letras, cores, formas geométricas, é natural admitir que os sistemas semióticos criados pelos códigos 
tecnológicos se encarregaram de gerar variáveis semióticas de outra natureza. Visualização do espaço, mapas semânticos, culturalização do espaço: eis algumas das variáveis que compõem o repertório da mudança e de suas práticas rumo a uma assimilação dos vetores do espaço ressonante cuja ação não deixou imune nem mesmo a consciência geopolítica agora modelizada pelos signos da tecnocultura.

Irene Machado é professora livre-docente em Ciências da Comunicação pela Universidade de São Paulo, Escola de Comunicações e Artes. Atua no Programa de PósGraduação em Meios e Processos Audiovisuais da USP. É bolsista Produtividade CNPq (PQ-1D).

irenemac@uol.com.br

\section{Referências}

BODENHAMER, D. The Potential of Spacial Humanities. In: BODENHAMER, D.; CORRIGAN, J.; HARRIS, T. M. (eds.).The Spatial Humanities. GIS and the Future of Humanities Scholarship. Bloomington \& Indianápolis: Indiana University Press, 2010.

ERLMANN, V. Reason and Resonance. A History of Modern Aurality. Cambridge: MIT Press, 2010. FERRARA, L. D'A. Percepção ambiental, informação e contextualização. Sinopses, 1990.

. Design em espaços. São Paulo: Rosari, 2002.

HALL, E. T. A dimensão oculta. Tradução de Sonia Coutinho. Rio de Janeiro: Francisco Alves, 1977. HOUTUM, H. V. The geopolitics of borders and boundaries, Geopolitics, 10: 672-679, 2005.

JAKOBSON, R. Decadência do cinema? In: Linguística. Poética. Cinema. Tradução de Haroldo de Campos. São Paulo: Perspectiva, 1970.

LÓTMAN, I. A estrutura do texto artístico. Lisboa: Estampa, 1978.

Universe of the Mind. A Semiotic Theory of Culture. Tradução de Ann Shukmann. Bloomington: Indiana University Press, 1990.

La semiosfera. L'asimmetria e il dialogo nelle struture pensanti. Ed. eTradução de Simonetta Salvestroni. Venezia: Marsilio, 1985.

El texto y el poliglotismo de la cultura. In: La semiosfera. Semiotica de la cultura y del texto. Tradução de Desidério Navarro. Madrid: Catedra, 1996.

MCLUHAN, M.; POWERS, B. R. Exploraciones en el espacio visual y el acústico. In: McLUHAN, M. La aldea global. Barcelona: Gedisa, 1996.

MACHADO, I. Língua entre linguagens: a argumentação gráfica na comunicação da ciência. São Paulo: Universidade de São Paulo, 2011. (Tese de Livre Docência)

Geopolítica dos espaços de informação: percepção, ambiente, ontologia. Anais do XXI Encontro Anual da COMPÓS, Juiz de Fora, 2012. www.compos.br/biblioteca 
Espaços semióticos modelizados pelos meios. Anais do XXI Encontro Anual da COMPÓS, Belém do Pará, 2014a. www.compos.br/biblioteca

. Variáveis semióticas do espaço na cultura de meios. GP Semiótica da comunicação. XXXVII Congresso Brasileiro de Ciências da Comunicação. Intercom, Foz do Iguaçu, 2014b.

. Espaço geopolítico em tradução semiótica. XII Congresso da Associação Internacional de Lusitanistas, Cabo Verde: Universidade de Mindelo, 2014c.

MAGNOLI, D. O que é geopolítica. São Paulo: Brasiliense; Círculo do Livro, 1990.

ONG, W. Jr. The Presence of the Word. Studies in the Evolution of Consciousness and Culture. Ithaca and London: Cornell University Press, 1967.

PRICE, M. E. Media and Sovereignty. Cambridge: MIT Press, 2002.

SANTOS, M. A natureza do espaço. São Paulo: Edusp, 2006.

SCHLÖGEL, K. En el espacio leemos el tiempo. Sobre história de la civilización y geopolítica. Tradução de José Luis Arántegui. Madrid: Siruela, 2007.

SEBEOK, T. Comunicação. In: NEIVA, E.; RECTOR, M. (orgs.). Comunicação na era pós-moderna. Rio de Janeiro: Vozes, 1995.

STERNE, J. The Audible Past. Cultural Origins of Sound Reproduction. Durham; London: Duke University Press, 2003.

UEXKÜLL, J. V. An Introduction to Umwelt. Semiotica, 134, 1/4, p. 107-110, 2001.

Artigo recebido em setembro

e aprovado em outubro de 2014. 